Series A

I. MATHEMATICA

592

\title{
ON THE FIRST COEFFICIENT REGIONS OF BOUNDED UNIVALENT FUNCTIONS
}

BY

RONALD KORTRAM and OLLI TAMMI

HEL I N K I 1974

S U O M A L A I EN TIEDEAKATEMIA

doi:10.5186/aasfm.1975.592 
Copyright (C) 1974 by

Academia Scientiarum Fennica

ISSN 0066-1953

ISBN 951-41-0208.8

Communicated 9 April 1974

KESKUSKIRJAPAINO

HELSINKI 1975 


\section{Introduction}

Let $S$ be the class of univalent functions $F$ on the unit disc normalized in such a way that

$$
F(z)=z+\sum_{n=2}^{\infty} a_{n} z^{n},
$$

and let for any number $b \in(0,1], S(b)$ be the class of univalent functions $f$ on the unit disc

$$
f(z)=b\left\{z+\sum_{n=2}^{\infty} a_{n} z^{n}\right\}
$$

for which $|f(z)|<1$. The set $V_{n+1} \subset C_{n}$ which is obtained by assigning to each function $F \in S$ (respectively $f \in S(b))$ the point $\left(a_{2}, a_{3}, \ldots, a_{n+1}\right)$ is called the $n$-th coefficient region. Schaeffer and Spencer [3] obtained $V_{3}$ for the class $S$ while Charzyński and Janowski [1] studied the case of $S(b)$. In [1] as well as in [3] the authors used variational methods.

The subclasses $S_{R} \subset S$ and $S_{R}(b) \subset S(b)$ consisting of functions with real coefficients are easier to handle. Already in 1936, Peschl [2] obtained by methods similar to Löwner's parametric method the shape of $V_{3}$ for the case of $S_{R}$.

In this paper we want to determine the functions corresponding to the boundary of $V_{3}$. Therefore we use an area integration method which enables us to prove the uniqueness of the extremal functions. We also use Löwner methods, which, however, do not confirm the expected uniqueness of the extremals. For $S_{R}(b)$ we determine $V_{3}$ completely while for $S(b)$ we find only a part of $V_{3}$. In the case of $S_{R}(b)$ we also determine a part of the region $V_{4}$.

\section{The Power inequalities}

The main tool in our investigations is the $P_{N}$ inequality derived in [4]. In order to make the notation clear we recall some definitions. Let $f \in S(b)$,

The first author is supported by the Netherlands Organization for the advance. ment of Pure Research (Z.W.O.). 
A. I. 592

let $x_{0}$ be a real parameter and let $x_{n}(n= \pm 1, \pm 2, \ldots)$ be complex parameters. Define the coefficients $c_{n k}$ by

$$
\left\{\begin{array}{l}
f(z)^{n}=\sum_{k=-\infty}^{\infty} c_{n k} z^{k} \quad(n \in Z, \quad n \neq 0), \\
\log \frac{f(z)}{z}=\sum_{k=-\infty}^{\infty} c_{o k} z^{k},
\end{array}\right.
$$

and let

$$
y_{k}=\sum_{n=-N}^{k} x_{n} c_{n k} \quad(k \geq-N) .
$$

These numbers satisfy the inequality $[4 ;$ page 5$]$

$$
2 x_{0} \operatorname{Re} y_{0}+\sum_{k=-N}^{\infty} k\left|y_{k}\right|^{2} \leq \sum_{k=-N}^{N} k\left|x_{k}\right|^{2} .
$$

We shall use the weaker form

$$
2 x_{0} \operatorname{Re} y_{0}+\sum_{k=-N}^{N} k\left|y_{k}\right|^{2} \leq \sum_{k=-N}^{N} k\left|x_{k}\right|^{2} .
$$

This is the $P_{N}$ inequality. Equality here is possible only if $y_{k}=0(k>N)$. This is equivalent to

$$
\sum_{k=N+1}^{\infty} y_{k} z^{k}=0,
$$

and it is easy to see furthermore that this is equivalent to

$$
x_{0} \log \frac{f(z)}{z}+\sum_{\substack{n=-\infty \\ n \neq 0}}^{N} x_{n} f(z)^{n}=\sum_{n=-N}^{N} y_{n} z^{n} .
$$

In view of later purposes we shall study relation (2.2) for some special cases. We shall show that for suitably chosen parameters (2.2) defines a bounded univalent function.

Take $N=1$, and impose the following restrictions on the parameters:

$$
0 \leq \frac{1}{2} x_{0} \leq x_{1}<y_{1} ; x_{-1}=-x_{1} ; y_{-1}=-y_{1}
$$

By the implicit function theorem the relation

$$
x_{0} \log \frac{f}{z}+x_{1}\left(f-f^{-1}\right)=y_{1}\left(z-z^{-1}\right)+y_{0}
$$

defines $f$ locally as an analytic function of $z$ for all values of $z$ for which 


$$
x_{1} f^{2}+x_{0} f+x_{1} \neq 0 \text {. }
$$

(To study $f$ in a neighbourhood of $z=0$ we investigate the function $g$ given by $f=x_{1} z / y_{1}+z^{2} g$. This explains why we require that $\left.x_{1}<y_{1}\right)$. In order to find the points $z$ for which (2.3) becomes zero, write $z=\varrho e^{i \prime \prime}$, $f=r e^{i \varphi}$, and split $\left(2.2^{\prime}\right)$ into its real and imaginary part. We obtain

$$
\left\{\begin{array}{l}
x_{0} \log r+x_{1}\left(r-r^{-1}\right) \cos \varphi=x_{0} \log \varrho+y_{1}\left(\varrho-\varrho^{-1}\right) \cos \vartheta+\operatorname{Re} y_{0}, \\
x_{0} \varphi+x_{1}\left(r+r^{-1}\right) \sin \varphi=x_{0} \vartheta+y_{1}\left(\varrho+\varrho^{-1}\right) \sin \vartheta+\operatorname{Im} y_{0} .
\end{array}\right.
$$

A necessary condition to obtain a circular slit domain is that all points $f$ with $r=1$ satisfy (2.4). Therefore we require that $\operatorname{Re} y_{0}=0$. The points $f$ for which (2.3) is zero are not real (because $0 \leq x_{0} \leq 2 x_{1}$ ), and have modulus one. Denote them by $e^{i \varphi_{0}}$ and $e^{-i \varphi_{0}}$. Substitution in (2.4) gives

$$
\left\{\begin{array}{l}
x_{0} \log \varrho+y_{1}\left(\varrho-\varrho^{-1}\right) \cos \vartheta=0, \\
x_{0} \vartheta+y_{1}\left(\varrho+\varrho^{-1}\right) \sin \vartheta+\operatorname{Im} y_{0}=x_{0} \varphi_{0}+2 x_{1} \sin \varphi_{0} .
\end{array}\right.
$$

The first equation represents a curve which has the shape indicated in Figure la. The position of $z$ on this curve is determined by the second
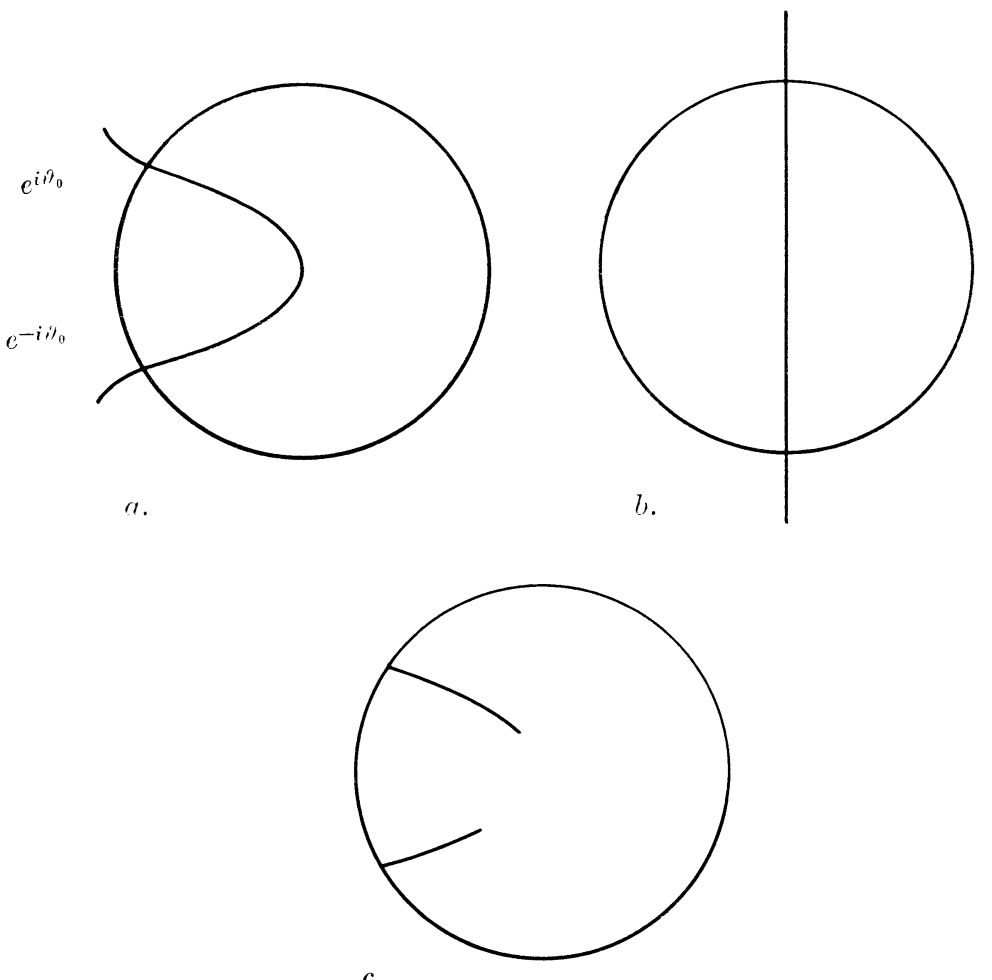

Figure 1. 
equation. In order to find a point $z=\varrho e^{i \vartheta}$ with $\varrho=1$ for which $(2.3)$ becomes zero it is necessary and sufficient that the equation

$$
x_{0} \vartheta+2 y_{1} \sin \vartheta+\operatorname{Im} y_{0}=x_{0} \varphi_{0}+2 x_{1} \sin \varphi_{0}
$$

has a solution. Because of (2.3) $\varphi_{0}$ satisfies the equation

$$
2 x_{1} \cos \varphi_{0}+x_{0}=0 \text {. }
$$

This means that (2.5) has a solution if the projection of the graph of $x_{0} \vartheta+2 y_{1} \sin \vartheta+\operatorname{Im} y_{0}$ on the vertical axis covers the projection of the graph of $x_{0} \varphi+2 x_{1} \sin \varphi$. This condition on $\operatorname{Im} y_{0}$ is certainly satisfied if $y_{0}=0$. If this condition is fulfilled, then we see that $f$ defined by $\left(2.2^{\prime}\right)$ is analytic at every point of the unit disc and by monodromy $f$ is singlevalued for $|z|<1$. In order to show that $f$ is univalent and bounded we study its boundary behaviour. From (2.4) we obtain for $\varrho=1$ that the image of the unit circle is contained in the locus of the curve

$$
x_{0} \log r+x_{1}\left(r-r^{-1}\right) \cos \varphi=0 .
$$

The shape of this curve is similar to that of Figure 1a. The branch points have in this case a real part which is more negative. The position of $f(z)$ on the curve is given by the second equation of (2.4). Until the points $\pm \varphi_{0}$ are reached, $f(z)$ moves along the unit circle. Then $f(z)$ travels along a slit until $\vartheta$ has reached $\pm \vartheta_{0}$ (cf. Figure 2), whereafter $f(z)$ goes back to the circle and continues its path along the circle. This shows that $f$ is bounded and univalent. To show that $f \in S\left(x_{1} y_{1}\right)$ it is sufficient to substitute a power series in (2.2).

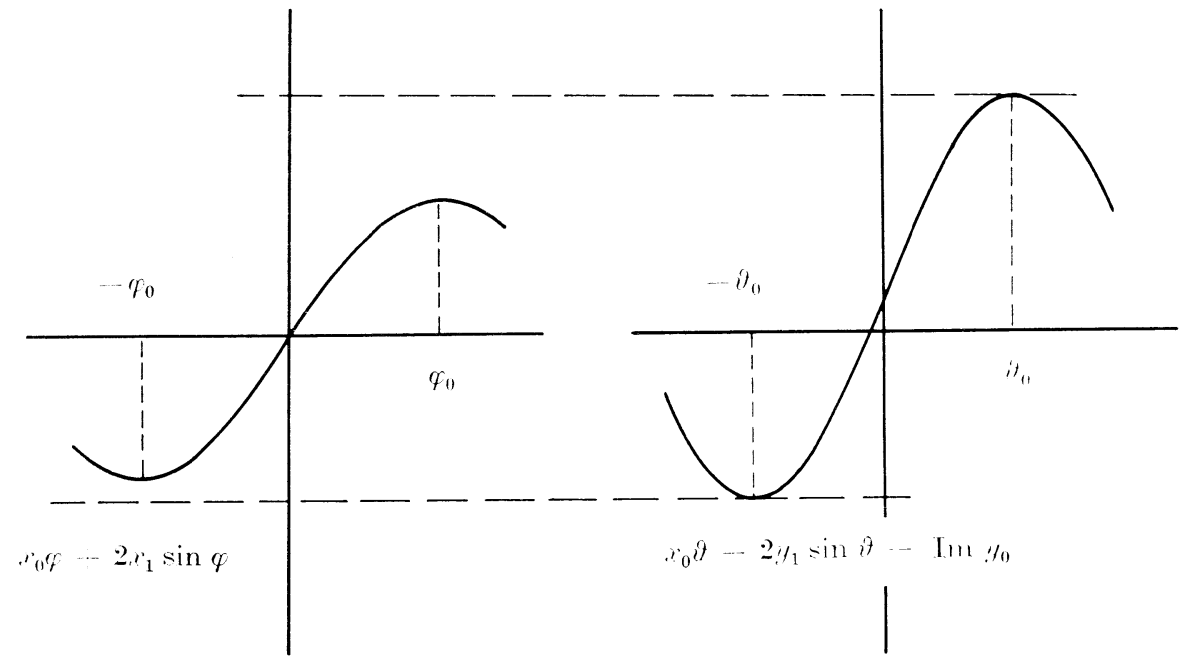

Figure 2. 
If $x_{0}=0$, then the locus of the first equation of (2.4) reduces to the curve shown in Figure 1b. The image domain is in this case a circle with two rectilinear slits. The length of the slits is determined by $y_{0}$. In all cases $\left(x_{0}=0, x_{0} \neq 0\right)$ the slits will have equal length if $y_{0}=0$. In Figure 1c a sketch of the image domain is given for the case $y_{0}>0, x_{0} \neq 0$.

\section{Löwner's parametric method}

In order to derive bounds for $a_{3}$ as a function of $a_{2}$ we base our investigations in the case of $S_{R}(b)$ on Löwner's theory for symmetric functions [5]. We shall use the following results [5; page $9-10$ ].

Consider schlicht two-slit domains $B$ consisting of the unit disc with two symmetric Jordan curves (omitting the origin) as slits. For every such domain $B$ there is a continuous function $\vartheta(u)$ on an interval $[b, 1]$ with the property: when

$$
f(z, u)=u\left\{z+\sum_{\nu=2}^{\infty} a_{\nu}(u) z^{\nu}\right\}, \quad|z|<1,
$$

denotes the solution of the equation

$$
u \frac{\partial f(z, u)}{\partial u}=\frac{f(z, u)-f(z, u)^{3}}{1-2 \cos \vartheta(u) f(z, u)+f(z, u)^{2}}
$$

and satisfies the initial condition $f(z, 1)=z$, then $f(z, u)$ contains the mapping function giving $B$ for $u=b$. Because of the symmetry of the domain $B$ the coefficients $a_{v}(u)$ are real. Conversely, for every function $\vartheta(u)$ continuous on an interval $b \leq u \leq 1$, the solutions (3.1) of the equation (3.2) are univalent and bounded: $|f(z, u)|<1$ (and obviously they have real coefficients) provided the initial condition $f(z, 1)=z$ is fulfilled.

For the coefficients $a_{2}$ and $a_{3}$ we have the following expressions

$$
\left\{\begin{array}{l}
a_{2}=-2 \int_{b}^{1} \cos \vartheta(u) d u, \\
a_{3}=4\left(\int_{b}^{1} \cos \vartheta(u) d u\right)^{2}-2 \int_{b}^{1} u \cos 2 \vartheta(u) d u .
\end{array}\right.
$$

If we use stepfunctions instead of continuous functions $\vartheta(u)$, the set of solutions of (3.2) also exhausts the class $S_{R}(b)$.

The problem of finding a lower bound for $a_{3}$ can be solved directly. From (3.3) we see that 


$$
a_{3}=a_{2}^{2}-2 \int_{b}^{1} u \cos 2 \vartheta(u) d u
$$

and this implies that

$$
a_{3} \geq a_{2}^{2}-2 \int_{b}^{1} u d u=a_{2}^{2}-\left(1-b^{2}\right)
$$

Equality is possible if $\cos 2 \vartheta(u)=1$ for $u \in[b, 1]$. By taking $\vartheta(u)=0$ on suitably chosen intervals and $\vartheta(u)=\pi$ on the remaining part of $[b, 1]$ we can arrange that $a_{2}$ has a preassigned value, so (3.4) is sharp.

An upper bound can be obtained similarly:

$$
a_{3}=a_{2}^{2}-2 \int_{b}^{1} u \cos 2 \vartheta(u) d u=a_{2}^{2}+\left(1-b^{2}\right)-4 \int_{b}^{1} u \cos ^{2} \vartheta(u) d u,
$$

so

$$
a_{3} \leq a_{2}^{2}+\left(1-b^{2}\right)-4 \min \int_{b}^{1} u \cos ^{2} \vartheta(u) d u
$$

By Schwarz' inequality we have

$$
\left(\int_{b}^{1} \cos \vartheta(u) d u\right)^{2} \leq \int_{b}^{1} \frac{d u}{u} \int_{b}^{1} u \cos ^{2} \vartheta(u) d u
$$

hence

$$
4 \int_{b}^{1} u \cos ^{2} \vartheta(u) d u \geq-\frac{a_{2}^{2}}{\log b}
$$

Therefore

$$
a_{3} \leq 1-b^{2}+a_{2}^{2}\left(1+\frac{1}{\log b}\right) .
$$

The equality sign in Schwarz' inequality can only hold if $\cos ^{2} \vartheta(u)=\sigma^{2} / u^{2}$ for a suitably chosen constant $\sigma$ (with $|\sigma| \leq b$ ). The condition that $a_{2}$ is prescribed can be satisfied only if we can find $|\sigma| \leq b$ such that

$$
2 \int_{b}^{1} \frac{|\sigma|}{u} d u=\left|a_{2}\right|
$$

i.e. if $\left|a_{2}\right| \leqq-2 b \log b$. 


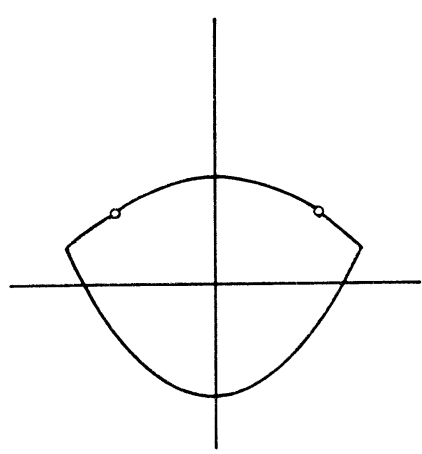

$e^{-1} \cdots b \cdots 1$

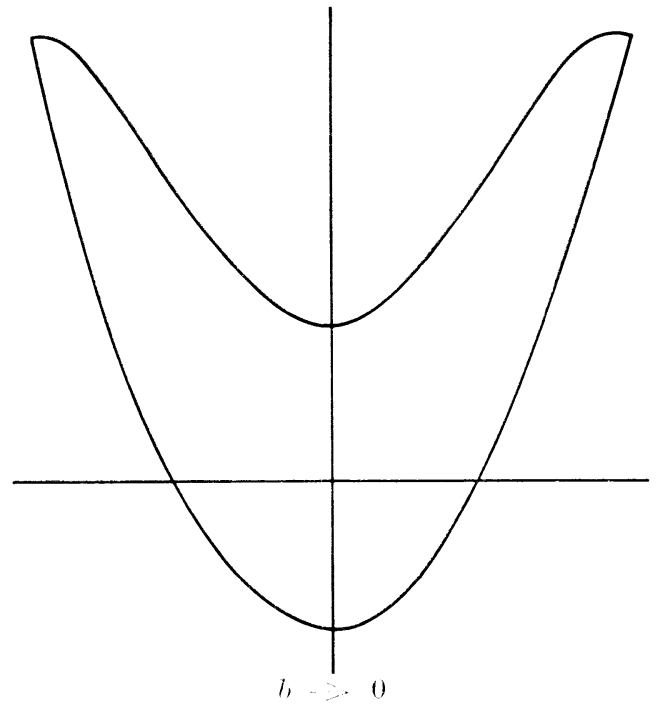

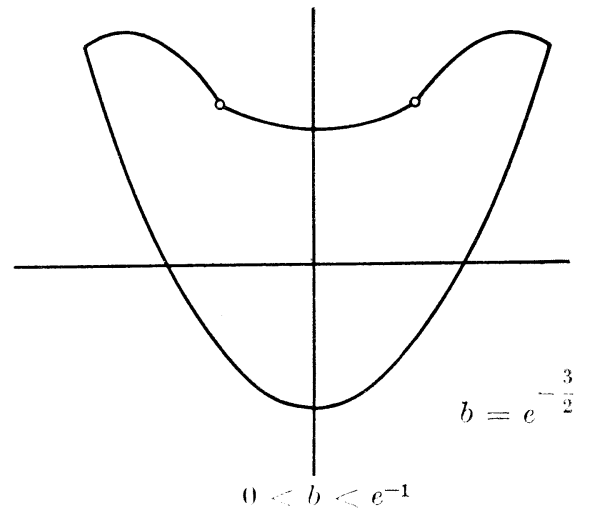

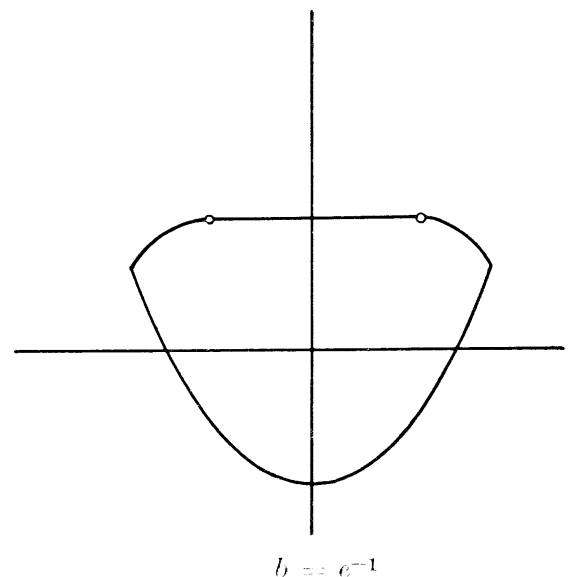

Figure 3.

If $\left|a_{2}\right|>-2 b \log b$ we cannot choose this function $\vartheta(u)$. From the identity (obtained by partial integration)

$$
\int_{b}^{1} u \cos ^{2} \vartheta(u) d u=b \int_{b}^{1} \cos ^{2} \vartheta(u) d u+\int_{b}^{1} d t \int_{t}^{1} d u \cos ^{2} \vartheta(u)
$$

we see that by prescribed value for $\int_{b}^{1} \cos \vartheta(u) d u$ the left-hand side is minimal if $\cos ^{2} \vartheta$ is decreasing. Hence take $\cos ^{2} \vartheta(u)=1$ on an interval $[b, \sigma]$ and $\cos ^{2} \vartheta=\sigma^{2} / u^{2}$ on $(\sigma, 1] . \quad \sigma$ has to be chosen so that $a_{2}$ has the prescribed value. This leads to 


$$
a_{3} \leq a_{2}^{2}+1-b^{2}-2\left(\sigma^{2}-b^{2}\right)+4 \sigma^{2} \log \sigma,
$$

where $\sigma$ is determined by

$$
\sigma-\sigma \log \sigma=b+\frac{1}{2}\left|a_{2}\right| .
$$

From (3.4), (3.5) and (3.6) we obtain the shape for $V_{3}$ indicated in Figure 3.

\section{The extremal functions}

The functions corresponding to the lower bound for $a_{3}$ and those corresponding to the upper bound for values of $a_{2}$ with $\left|a_{2}\right| \leq-2 b \log b$ shall be determined in Section 6. There we are able to show that they are uniquely determined. In order to obtain extremal functions for $\left|a_{2}\right|>-2 b \log b$ we integrate Löwner's equation (3.2). Therefore we have to substitute $\vartheta$, and obtain

$$
\left\{\begin{array}{l}
u \frac{\partial f(z, u)}{\partial u}=f(z, u) \frac{1+f(z, u)}{1-f(z, u)}, u \in[b, \sigma] ; \\
u \frac{\partial f(z, u)}{\partial u}=\frac{f(z, u)-f(z, u)^{3}}{1-\frac{2 \sigma}{u} f(z, u)+f(z, u)^{2}}, u \in[\sigma, 1] .
\end{array}\right.
$$

Note that the second equation is linear with respect to $u$. Integration of the equation with the initial condition $f(z, 1)=z$ gives

$$
\begin{gathered}
\frac{f(z, u)}{(1+f(z, u))^{2}}=\frac{u}{\sigma} \frac{f(z, \sigma)}{(1+f(z, \sigma))^{2}}, u \in[b, \sigma] ; \\
u\left\{f(z, u)-f(z, u)^{-1}\right\}=z-z^{-1}+2 \sigma \log \frac{f(z, u)}{z}, u \in[\sigma, 1] .
\end{gathered}
$$

From our considerations in Section 2 it follows that (4.3) determines for $u=\sigma$ a function $f$ with image domain as in Figure 4a. Applying $f(z, b)$ defined by (4.2) we end up to the final image domain (Figure $4 b$ ).
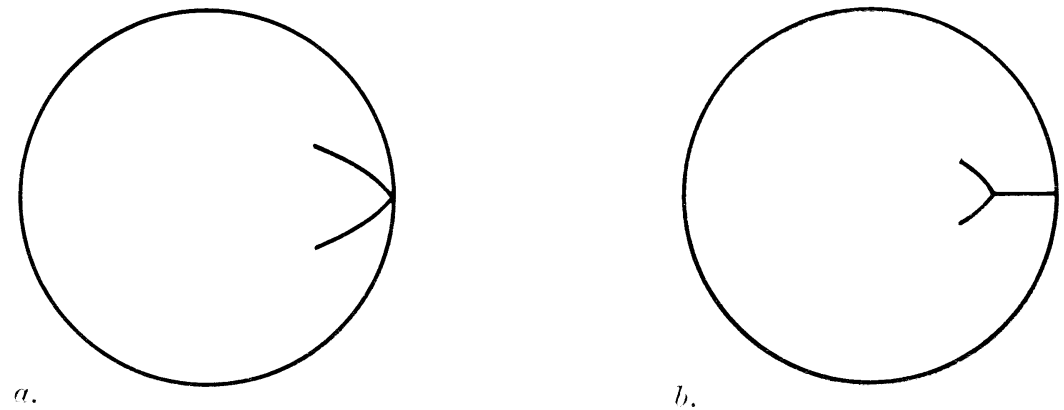

Figure 4 . 


\section{The region $\mathbf{V}_{4}$}

For functions $f \in S_{R}(b)$ we shall determine a part of the region $V_{4}$. We already know $V_{3}$. We use the $P_{3}$ inequality with the parameters $[6$; page 4,5$]$ :

$$
\left\{\begin{array}{l}
x_{3}=-x_{-3}=\frac{1}{3} b^{1 / 2} \\
x_{2}=-x_{-2}=0 \\
x_{1}=-x_{-1}=b^{1 / 2}\left\{\frac{1}{2} a_{2}+\frac{a_{3}-\frac{3}{4} a_{2}^{2}+b a_{2}}{2(1-b)-a_{2}}\right\},
\end{array}\right.
$$

and apply it to $h(z)=\sqrt{f\left(z^{2}\right)}$. After simple rearrangements we obtain

$$
a_{4} \leq \frac{2}{3}\left(1-b^{3}\right)+2 a_{2} a_{3}-\frac{13}{12} a_{2}^{3}-\frac{1}{2} b a_{2}^{2}-\frac{\left(a_{3}-\frac{3}{4} a_{2}^{2}+b a_{2}\right)^{2}}{2(1-b)-a_{2}} .
$$

Condition $(2.2)$ is in this case

$$
\begin{aligned}
& \frac{1}{3} b^{3 / 2}\left(h^{3}-h^{-3}\right)+b^{1 / 2} s\left(h-h^{-1}\right)=\frac{1}{3}\left(z^{3}-z^{-3}\right)+u\left(z-z^{-1}\right) \\
& \left\{\begin{array}{l}
u=\frac{a_{3}-\frac{3}{4} a_{2}^{2}+b a_{2}}{2(1-b)-a_{2}} \\
s=u+\frac{a_{2}}{2} .
\end{array}\right.
\end{aligned}
$$

Differentiation gives

$$
\begin{gathered}
b^{3 / 2} z \frac{h^{\prime}}{h}=\frac{z^{3}+z^{-3}+u\left(z+z^{-1}\right)}{h^{3}+h^{-3}+s\left(h+h^{-1}\right) / b}=\frac{h^{3}}{z^{3}} \frac{\left(z^{2}+1\right)\left(z^{2}-z_{1}^{2}\right)\left(z^{2}-z_{2}^{2}\right)}{\left(h^{2}+1\right)\left(h^{2}-h_{1}^{2}\right)\left(h^{2}-h_{2}^{2}\right)} \\
z_{12}= \pm \frac{\sqrt{3-u}}{2}+\frac{\sqrt{-u-1}}{2} \\
h_{12}= \pm \frac{\sqrt{3-s / b}}{2}+\frac{\sqrt{-s / b-1}}{2}
\end{gathered}
$$

From this we read that $z_{1}, z_{2}$ and $h_{1}, h_{2}$ are unit roots exactly if

$$
\begin{aligned}
& -1 \leq u \leq 3, \\
& -1 \leq s / b \leq 3 .
\end{aligned}
$$

The factorized differential equation suggests $h(U)$ to be a disc with six slits or with two three-fork slits. This can be studied more exactly by aid 


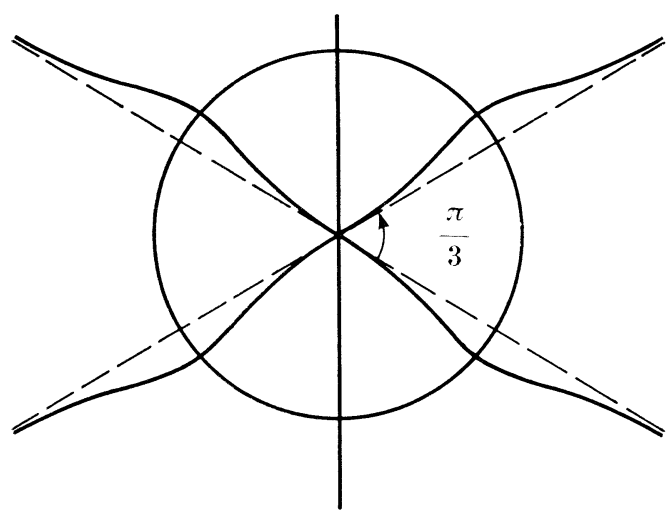

a. $-b \leq s \leq 3 b$

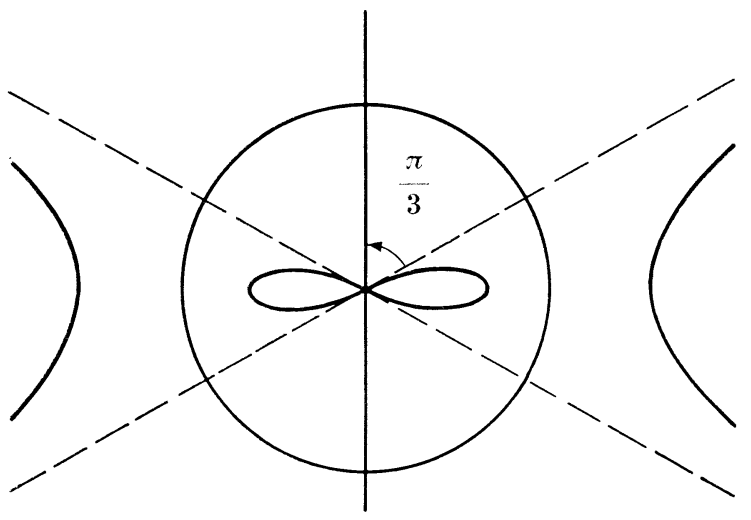

c. $s<\cdots b$

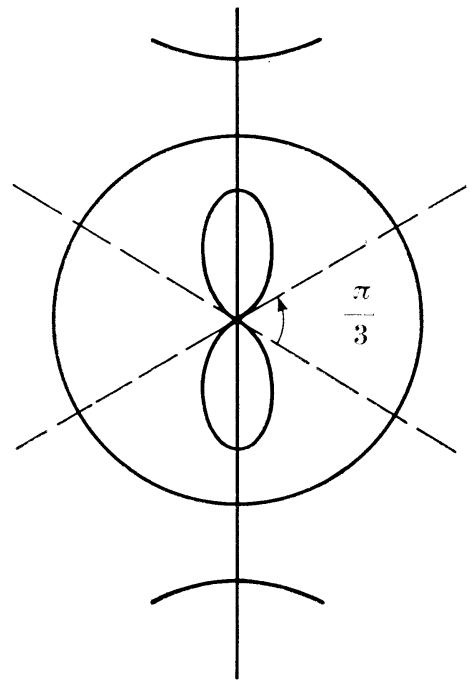

1). $\therefore 3 !$

Figure 5.

of the boundary correspondence $h=r e^{i \varphi}, z=e^{i \vartheta}$. Under this notation split (5.2) into real and imaginary parts. This leads to conditions:

$$
\frac{1}{3} b\left(r^{3}-r^{-3}\right) \cos 3 \varphi+s\left(r-r^{-1}\right) \cos \varphi=0,
$$

$$
\frac{1}{3} b^{3 / 2}\left(r^{3}+r^{-3}\right) \sin 3 \varphi+b^{1 / 2} s\left(r+r^{-1}\right) \sin \varphi=\frac{2}{3} \sin 3 \vartheta+2 u \sin \vartheta .
$$

The locus of equation (5.5) is indicated in Figure 5. Necessary, for existing of a $S(b)$-mapping defined by (5.2) is thus 


$$
-1 \leq s / b \text { and } u \leq 3
$$

To find sufficient conditions we study (5.6) and require that for $r=1$ each $\varphi \in[0,2 \pi]$ gets an uniquely determined pre-image $\vartheta \in[0,2 \pi]$. Comparing the graphs of the left and the right side we end up with the requirements

$$
\frac{1}{3}(u+1)^{3 / 2} \geq \frac{b^{3 / 2}}{3}(s / b+1)^{3 / 2}
$$

and

$$
-\frac{1}{3} b^{3 / 2}+b^{1 / 2} s \geq-\frac{1}{3}+u
$$

The first condition is equivalent to $2(1-b) \geq a_{2}$ and is thus always satisfied. The second gives a restriction

$$
a_{3} \leq \frac{1}{4} \frac{3-5 b^{1 / 2}}{1-b^{1 / 2}} a_{2}^{2}-\frac{1}{3}\left(1-b^{1 / 2}\right)^{2} a_{2}+\frac{2}{3}\left(1+b^{1 / 2}\right)\left(1-b^{3 / 2}\right)
$$

which implies $u \leq 3$. Thus (5.8) and the first condition (5.7) are the limitations which in the coefficient body define a domain where (5.1) is sharp.

In Figure 6 are given the types of the extremal domains $f(U)$ found. Figure 7 illustrates for $b=\frac{1}{2}$ the part of the coefficient body, where these extremum domains belong to. Horizontal lining and a. as well as the dotted domain and $\mathrm{b}$. belong together.

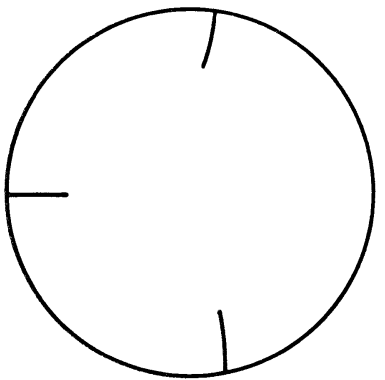

$a$.

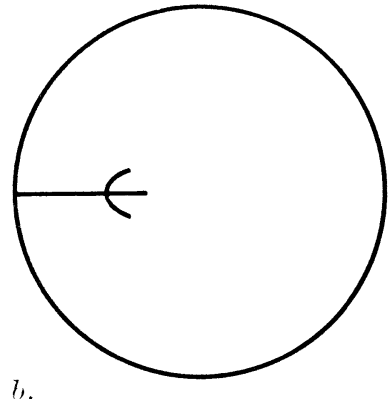

b.

Figure 6 . 


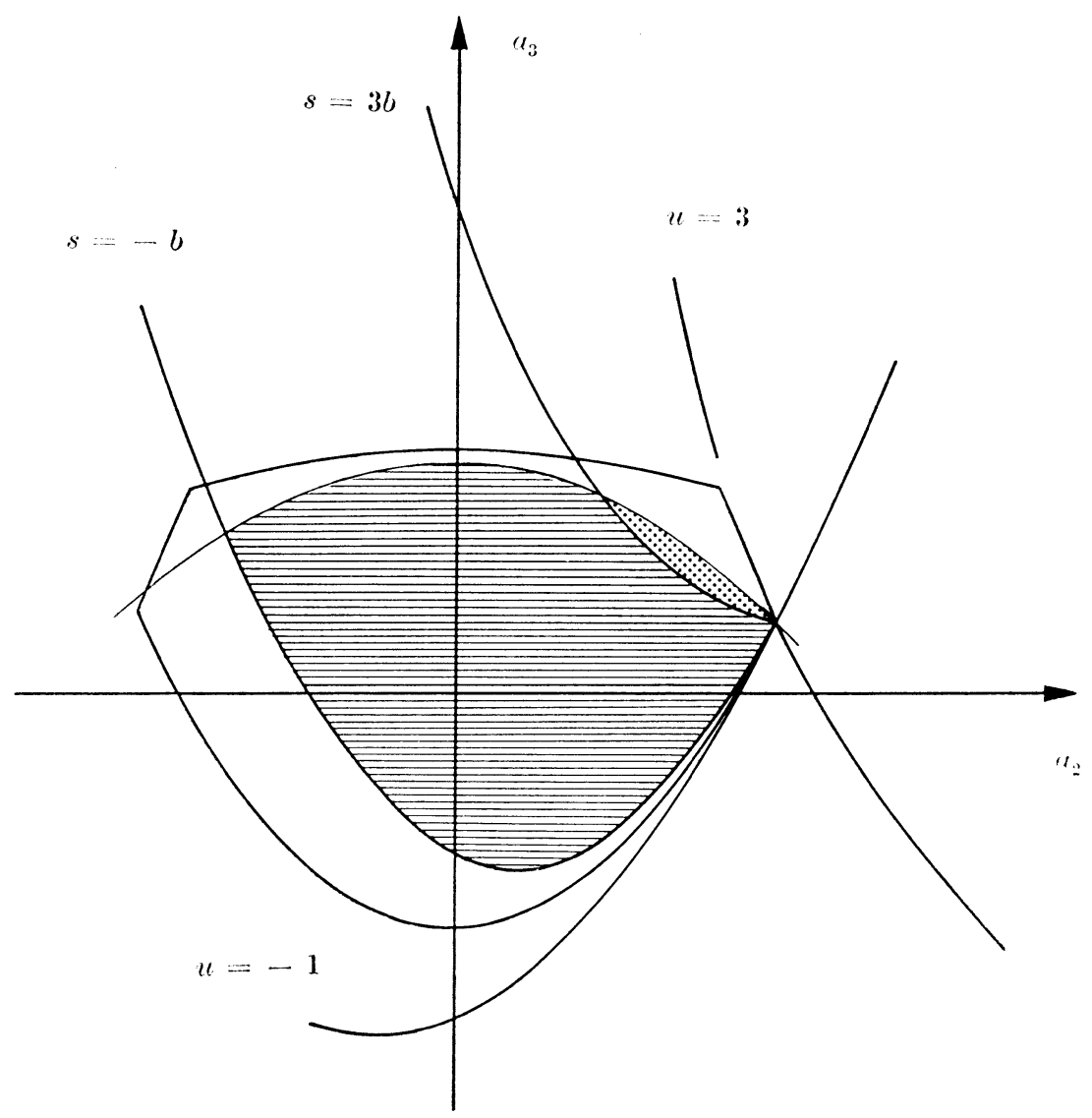

Figuge 7 .

\section{6. $P_{1}$ in $S_{R}(b)$}

A simple calculation gives for $f \in S(b)$ the following results:

$$
\left\{\begin{array}{l}
y_{-1}=\frac{x_{-1}}{b}, \\
y_{0}=x_{0} \log b-\frac{x_{-1}}{b} a_{2}, \\
y_{1}=\frac{x_{-1}}{b}\left(a_{2}^{2}-a_{3}\right)+x_{0} a_{2}+x_{1} b .
\end{array}\right.
$$

In accordance with $\left[6\right.$; page 5] choose $u_{1}=-y_{-1}$ as a new parameter. We have 


$$
\left\{\begin{array}{l}
y_{-1}=-u_{1} \\
y_{0}=x_{0} \log b+u_{1} a_{2}, \\
y_{1}=-u_{1}\left(a_{2}^{2}-a_{3}\right)+x_{0} a_{2}+x_{1} b .
\end{array}\right.
$$

In the case of $S_{R}(b)$ we choose all parameters real. The $P_{1}$ inequality is in this notation

$$
2 x_{0}^{2} \log b+2 x_{0} u_{1} a_{2}+\left\{\left(a_{3}-a_{2}^{2}\right) u_{1}+x_{0} a_{2}+x_{1} b\right\}^{2}-x_{1}^{2}-\left(1-b^{2}\right) u_{1}^{2} \leq 0 .
$$

We want to find the range of

$$
\delta=a_{3}-a_{2}^{2}
$$

and therefore we choose the parameters in such a way that the left-hand side of the $P_{1}$ inequality becomes maximal. There are two different cases, $x_{0}=0$ and $x_{0} \neq 0$.

First case: $x_{0}=0$. For $u_{1}=1$ the inequality reads

$$
\delta^{2}+2 \delta b x_{1}+b^{2} x_{1}^{2}-x_{1}^{2}-\left(1-b^{2}\right) \leq 0 .
$$

The left-hand side attains a maximum for $x_{1}=b \delta /\left(1-b^{2}\right)$, and (6.3) gives for this value of $x_{1}$

$$
\delta^{2}-\left(1-b^{2}\right)^{2} \leq 0
$$

giving us the important inequality

$$
|\delta| \leq 1-b^{2} .
$$

From (6.2) and (6.3) it can be seen that in the extremal case $\delta=-\left(1-b^{2}\right)$ and the parameters are equal to

$$
\left\{\begin{array} { l } 
{ x _ { 1 } = - b , } \\
{ x _ { 0 } = 0 , } \\
{ x _ { - 1 } = - b ; }
\end{array} \quad \left\{\begin{array}{l}
y_{1}=-1, \\
y_{0}=a_{2}, \\
y_{-1}=-1 .
\end{array}\right.\right.
$$

Condition (2.2) is in this case

$$
b\left(f+f^{-1}\right)=\left(z+z^{-1}-a_{2}\right) .
$$

In Figure 8 is indicated how the image domain of $f$ can be obtained.

From (6.2) and (6.1) one sees that in the extremal case $\delta=1-b^{2}$. The parameter values equal to

$$
\left\{\begin{array} { l } 
{ x _ { 1 } = b , } \\
{ x _ { 0 } = 0 , } \\
{ x _ { - 1 } = - b ; }
\end{array} \quad \left\{\begin{array}{l}
y_{1}=1, \\
y_{0}=a_{2}, \\
y_{-1}=-1 .
\end{array}\right.\right.
$$




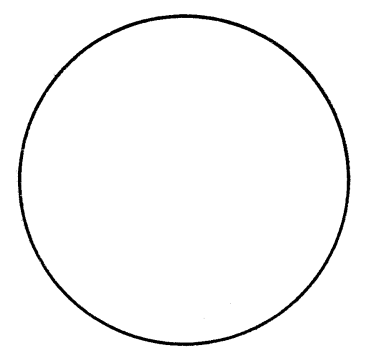

$\downarrow f(z)$

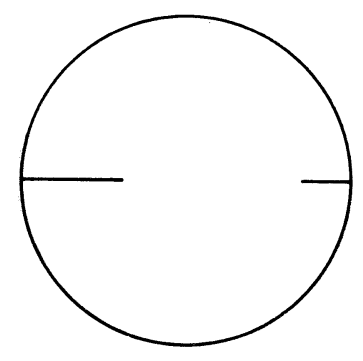

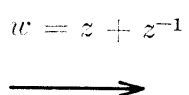

$-2$

2 $\zeta=\frac{1}{b}\left(w-a_{2}\right)$

Figure 8.

All conditions of Section 2 are satisfied except $\operatorname{Re} y_{0}=0$ unless $a_{2}=0$. Only in that case the upper bound $\delta=1-b^{2}$ can be reached. The image domain is the unit disc with two equal slits along the imaginary axis.

Second case: $x_{0} \neq 0$. Now we take $x_{0}=1$. The $P_{1}$ inequality is in this case

$$
\begin{gathered}
-\left(1-b^{2}\right) x_{1}^{2}-\left(1-b^{2}-\delta^{2}\right) u_{1}^{2}+2 b \delta x_{1} u_{1}+2 a_{2} b x_{1}+2 a_{2}(\delta+1) u_{1} \\
+2 \log b+a_{2}^{2} \leq 0 .
\end{gathered}
$$

By elementary calculations it can be shown that the quadratic function in $x_{1}$ and $u_{1}$ on the left side of this inequality attains its maximum for

$$
\left\{\begin{array}{l}
x_{1}=\frac{b a_{2}}{1-b^{2}-\delta} ; \delta \neq 1-b^{2}, \\
u_{1}=\frac{a_{2}}{1-b^{2}-\delta},
\end{array}\right.
$$


and for these values the inequality takes the form

$$
\left(\delta+1-b^{2}\right)\left\{\delta \log b-a_{2}^{2}-\left(1-b^{2}\right) \log b\right\} \geq 0 .
$$

In view of (6.4) we derive from this that

$$
\delta \leq 1-b^{2}+\frac{a_{2}^{2}}{\log b} .
$$

This is the result of (3.8) for the case that $\left|a_{2}\right| \leq-2 b \log b$. Again from (6.2) and (6.1) it follows that in the extremal case $\delta=1-b^{2}+a_{2}^{2} / \log b$ the parameters are equal to

$$
\left\{\begin{array} { l } 
{ x _ { 1 } = - \frac { b \operatorname { l o g } b } { a _ { 2 } } , } \\
{ x _ { 0 } = 1 , } \\
{ x _ { - 1 } = \frac { b \operatorname { l o g } b } { a _ { 2 } } ; }
\end{array} \left\{\begin{array}{l}
y_{1}=-\frac{\log b}{a_{2}} \\
y_{0}=0 \\
y_{-1}=\frac{\log b}{a_{2}} .
\end{array}\right.\right.
$$

The condition of Section $2, \frac{1}{2} x_{0} \leq x_{1}$, requires that $\left|a_{2}\right| \leq-2 b \log b$. For these values there exists an extremal function mapping the unit disc on a circular domain with two symmetric slits. The extremal function is determined by the equation

$$
\log \frac{f}{z}-\frac{b \log b}{a_{2}}\left(f-f^{-1}\right)=-\frac{\log b}{a_{2}}\left(z-z^{-1}\right) .
$$

This completes the results of Section 4 where the upper bound was derived in a different way, but where the discussion of the extremal functions was postponed.

\section{7. $P_{1}$ in $S(b)$}

Again we consider two cases, $x_{0}=0$ and $x_{0} \neq 0$. In the first case $x_{0}=0$ we can take $u_{1}=1$ and the inequality reads

$$
\left|\delta+x_{1} b\right|^{2} \leq\left|x_{1}\right|^{2}+1-b^{2}
$$

Denote $x_{1}=x+i y$ and $\delta=\delta_{1}+i \delta_{2}$. After simple rearrangements we obtain

$$
-\left(x-\frac{b \delta_{1}}{1-b^{2}}\right)^{2}-\left(y-\frac{b \delta_{2}}{1-b^{2}}\right)^{2}+\frac{|\delta|^{2}}{\left(1-b^{2}\right)^{2}}-1 \leq 0 .
$$

The optimal choice is thus 


$$
x_{1}=\frac{b \delta}{1-b^{2}},
$$

and this leads to

$$
|\delta| \leq 1-b^{2} .
$$

In the extremal case, $\delta=\left(1-b^{2}\right) e^{i \beta}$, the parameters are

$$
\left\{\begin{array} { l } 
{ x _ { 1 } = b e ^ { i \beta } , } \\
{ x _ { 0 } = 0 , } \\
{ x _ { - 1 } = - b ; }
\end{array} \quad \left\{\begin{array}{l}
y_{1}=e^{i \beta}, \\
y_{0}=a_{2}, \\
y_{-1}=-1 .
\end{array}\right.\right.
$$

Condition (2.2) reads

$$
b e^{i \beta} f(z)-b f(z)^{-1}=e^{i \beta} z-z^{-1}+a_{2} .
$$

Denote $w=e^{i \beta / 2} z$ and let $\tilde{f}(z)=e^{i \beta / 2} f\left(e^{-i \beta / 2} z\right)$. Then we have

$$
b\left(\tilde{f}-\tilde{f}^{-1}\right)=w-w^{-1}+a_{2} e^{-i \beta / 2} .
$$

The results of Section 2 can be applied to $\tilde{f}$. The conditions are

$$
\operatorname{Re} a_{2} e^{-i \beta / 2}=0,
$$

and

$$
-2(1-b) \leq \operatorname{Im} a_{2} e^{-i \beta / 2} \leq 2(1-b)
$$

The last one is always satisfied. The upper bound (7.1) is sharp if and only if $\arg \delta=2 \arg \left(i a_{2}\right)$. The image domain of $\tilde{f}$ is a disc with two (unequal) rectilinear slits along the imaginary axis. The image domain of $f$ is obtained from this by rotation over $-\beta / 2$.

In the second case where $x_{0} \neq 0$ the computations are more complicated. We take $x_{0}=1$ and split the parameters $x_{1}$ and $u_{1}$ and the quantities $a_{2}$ and $\delta$ in their real and imaginary parts, to write the lefthand side of the $P_{1}$ inequality in the form of a quadratic function of four variables $\operatorname{Re} x_{1}, \operatorname{Im} x_{1}, \operatorname{Re} u_{1}$ and $\operatorname{Im} u_{1}$. The parameters are again chosen in such a way that this function reaches an extremum. This is the case if

$$
\left\{\begin{array}{l}
x_{1}=-b \frac{a_{2}\left(1-b^{2}\right)+\bar{a}_{2} \delta}{\Delta} \\
u_{1}=-b \frac{\bar{a}_{2}\left(1-b^{2}\right)+a_{2} \bar{\delta}}{\Delta} \\
\Delta=|\delta|^{2}-\left(1-b^{2}\right)^{2}
\end{array}\right.
$$


The optimized $P_{1}$ inequality is then

$$
\Delta \log b-\operatorname{Re}\left(a_{2}^{2} \bar{\delta}\right)-\left(1-b^{2}\right)\left|a_{2}\right|^{2} \geq 0 .
$$

This is equivalent to

$$
\left|\delta-\frac{a_{2}^{2}}{2 \log b}\right| \leq R=1-b^{2}+\frac{\left|a_{2}\right|^{2}}{2 \log b} .
$$

Remark. $R$ is positive; this is a consequence of the inequality $\left|a_{2}\right| \leq 2(1-b)$ and the elementary fact that $2 y \leq \log [(1+y):(1-y)]$ for $0 \leq y<1$. Therefore (7.4) improves (7.1). This is shown in Figure 9 . In the extremal case we deduce from (7.3) that

$$
\Delta=\frac{\operatorname{Re}\left(a_{2}^{2} \bar{\delta}\right)+\left(1-b^{2}\right)\left|a_{2}\right|^{2}}{\log b} .
$$

From (7.2), (6.2) and (6.1) we infer that the parameters are

$$
\left\{\begin{array} { l } 
{ x _ { 1 } = - b \frac { a _ { 2 } ( 1 - b ^ { 2 } ) + \overline { a } _ { 2 } \delta } { \Delta } , } \\
{ x _ { 0 } = 1 , } \\
{ x _ { - 1 } = - \overline { x } _ { 1 } ; }
\end{array} \left\{\begin{array}{l}
y_{1}=\frac{x_{1}}{b}, \\
y_{0}=\log b-\frac{\left|a_{2}\right|^{2}\left(1-b^{2}\right)+a_{2}^{2} \bar{\delta}}{\Delta} \\
y_{-1}=-\bar{y}_{1} .
\end{array}\right.\right.
$$

Substitution of (7.5) in $y_{0}$ gives $\operatorname{Re} y_{0}=0$. It is convenient to use the notation

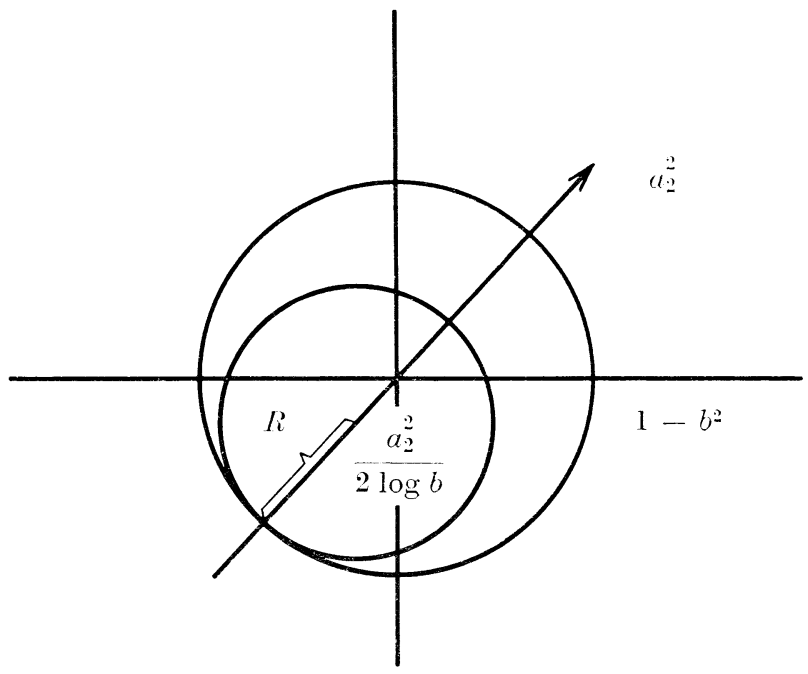

Figure 9. 


$$
\begin{gathered}
\left(1-b^{2}\right)\left|a_{2}\right|^{2}+a_{2}^{2} \bar{\delta}=x e^{i \omega} \quad(\varkappa>0), \\
a_{2}=\left|a_{2}\right| e^{i \alpha} .
\end{gathered}
$$

We see from this that

$$
\left\{\begin{array}{l}
\cos \omega=\frac{1}{x}\left\{\operatorname{Re}\left(a_{2}^{2} \bar{\delta}\right)+\left(1-b^{2}\right)\left|a_{2}\right|^{2}\right\}=\frac{1}{x} \Delta \log b \\
x=\left|a_{2}\right| \sqrt{2 R \Delta \log b} \quad\left(R=1-b^{2}+\frac{\left|a_{2}\right|^{2}}{2 \log b}\right) .
\end{array}\right.
$$

Because of (7.1) this shows that $\cos \omega \geq 0$. In this notation the condition (2.2) reads

$$
\begin{aligned}
& \log \frac{f}{z}+\frac{b \log b}{\left|a_{2}\right| \cos \omega}\left\{-e^{i(\alpha-\omega)} f+e^{-i(\alpha-(\omega)} f^{-1}\right\} \\
= & \frac{\log b}{\left|a_{2}\right| \cos \omega}\left\{-e^{i(\alpha-\omega)} z+e^{-i(\alpha-\omega)} z^{-1}\right\}-i \frac{\sin \omega}{\cos \omega} \log b .
\end{aligned}
$$

Denote $\zeta=z e^{i(\alpha-\omega)}$, and let $h(z)=e^{i(\alpha-\omega)} f\left(z e^{-i(\alpha-\omega)}\right)$. Then (7.7) becomes

$$
\begin{gathered}
\log \frac{h(\zeta)}{\zeta}+\frac{b|\log b|}{\left|a_{2}\right| \cos \omega}\left\{h(\zeta)-h(\zeta)^{-1}\right\}=\frac{\mid \log b}{\left|a_{2}\right| \cos \omega}\left\{\zeta-\zeta^{-1}\right\} \\
-i \frac{\sin \omega}{\cos \omega} \log b .
\end{gathered}
$$

The condition $\frac{1}{2} x_{0} \leq x_{1}$ of Section 2 prescribes that

$$
\frac{2 b|\log b|}{\left|a_{2}\right| \cos \omega} \geq 1
$$

and this is satisfied if

$$
\left|a_{2}\right| \leq-2 b \log b
$$

To study the condition on $\operatorname{Im} y_{0}$ we substitute

$$
t=\frac{\left|a_{2}\right| \cos \omega}{2 b \log b},
$$

and calculate the maximum and the minimum values of the functions $x_{0} \varphi+2 x_{1} \sin \varphi$ resp. $x_{0} \vartheta+2 y_{1} \sin \vartheta+\operatorname{Im} y_{0}$. The condition is

$$
\begin{aligned}
& F(t)=b\left(t \arccos t-\sqrt{1-t^{2}}\right)+\sqrt{1-b^{2} t^{2}}-b t \arccos b t \\
& -\frac{1}{2}\left|a_{2}\right| \sqrt{1-\frac{4 b^{2} \log ^{2} b}{\left|a_{2}\right|^{2}}} t^{2} \geq 0 \text { for } t \in\left[\frac{\left|a_{2}\right|}{2 b|\log b|}, 0\right] .
\end{aligned}
$$


Because $F(0)=1-b-\frac{1}{2}\left|a_{2}\right| \geq 0$, it is sufficient to prove that $F$ is decreasing on $\left[\left|a_{2}\right|: 2 b \log b, 0\right]$, i.e. $F^{\prime} \leq 0$. An easy calculation gives $F^{\prime}(0)=0$. It is therefore sufficient to prove that $F^{\prime}$ is increasing, i.e. $F^{\prime \prime} \geq 0$. We compute that

$$
\begin{gathered}
F^{\prime \prime}(t)=b\left(\frac{b}{\sqrt{1-b^{2} t^{2}}}-\frac{1}{\sqrt{1-t^{2}}}\right)+\frac{2 b^{2} \log ^{2} b}{\mid a_{2} !}\left(\frac{1}{\sqrt{1-\frac{4 b^{2} \log ^{2} b}{\left|a_{2}\right|^{2}} t^{2}}}\right)^{3} \geq \\
b\left\{\frac{b}{\sqrt{1-b^{2} t^{2}}}-\frac{1}{\sqrt{1-t^{2}}}\right\}+\frac{\log b \mid}{\left(\sqrt{1-t^{2}}\right)^{3}} .
\end{gathered}
$$

In this estimate we have used the inequality $\left|a_{2}\right| \leq-2 b \log b$. To show that $F^{\prime \prime}>0$ it suffices to show that

$$
\frac{b}{\sqrt{1-b^{2} t^{2}}} \geq \frac{1-t^{2}+\log b}{\left(\sqrt{1-t^{2}}\right)^{3}} .
$$

This is clearly the case if $t^{2} \geq 1+\log b$, so we can restrict ourselves to the case that $x=t^{2} \in[0,1+\log b]$ and we have to consider only values of $b$ with $b>e^{-1}$. Since both sides of (7.10) are non-negative we can square. Rearrangements lead to the inequality

$$
\begin{aligned}
& G(x)=x^{2}\left(b^{2}-1-2 b^{2} \log b\right) \\
& +x\left(2-2 b^{2}+2 \log b+b^{2} \log ^{2} b+2 b^{2} \log b\right)+b^{2}-(1+\log b)^{2} \geq 0 .
\end{aligned}
$$

Since $b^{2}-1-2 b^{2} \log b \leq 0$, it is sufficient to prove this inequality for $x=0$ and for $x=1+\log b$. It is easy to see that

$$
\begin{aligned}
& G(0)=b^{2}-(1+\log b)^{2} \geq 0, \\
& G(1+\log b)=-b^{2} \log ^{3} b \geq 0 .
\end{aligned}
$$

The function $h$ satisfies the conditions of Section 2 , hence its image domain is a disc with two (unequal) slits. The image domain of $f$ can be obtained from this by a simple rotation. In Figure 10 we have indicated the shape of this image domain for some special cases.

Condition (7.8) allows greater freedom for $a_{2}$ than condition (7.8'). However, we are not able to take full advantage of this fact. From (7.6) we deduce that in the extremal case

$$
\cos \omega=\frac{1}{\left|a_{2}\right|} \sqrt{\frac{\Delta \log b}{2 R}} .
$$

Substitution in (7.8) gives

$$
-\Delta \leq-8 b^{2} R \log b,
$$

i.e. 

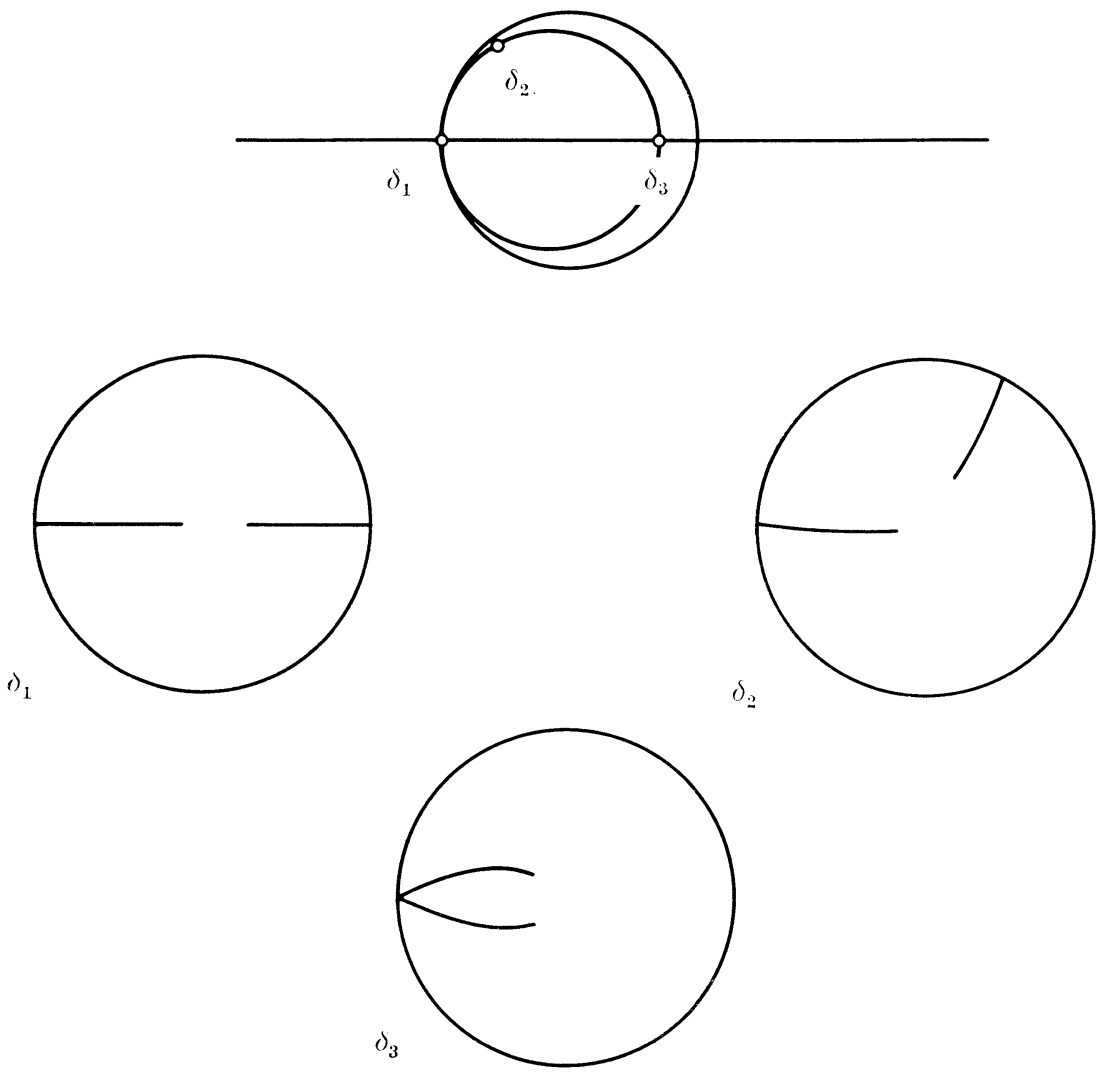

$$
\left\{\begin{array}{l}
\delta_{1}=-\left(1-e^{-2}\right) \\
\delta_{2}=-\frac{1}{2}\left(1+e^{-2}\right)+\frac{1}{2} i \sqrt{3}\left(1-3 e^{-2}\right) \\
\delta_{3}=-1-5 e^{-2}
\end{array}\right.
$$

Figure 10.

$$
|\delta|^{2} \geq\left(1-b^{2}+4 b^{2} \log b\right)^{2}+4 b^{2}\left(\left|a_{2}\right|^{2}-4 b^{2} \log b\right) .
$$

Comparison with (7.4) shows that for $\left|a_{2}\right|>-2 b \log b$ an arc of the bounding circle of (7.4) is always excluded.

The conditions of Section 2 are satisfied if the function (7.9) is nonnegative for $t \in[-1,0]$. By numerical computations one can show that this is not always the case if $\left|a_{2}\right|>-2 b \log b$. From those numerical computations it follows that for certain combinations $a_{2}, \omega$ we obtain additional information about the coefficient region. The extremal domains corresponding to the additional part are two-slit domains. 


\section{Corollaries}

It is easy to derive estimates concerning $a_{3}$ from the results we have for $\delta$. From (7.4) we see that $a_{3}$ lies in the disc

$$
\left|a_{3}-\left(1+\frac{1}{2 \log b}\right) a_{2}^{2}\right| \leq R=1-b^{2}+\frac{\left|a_{2}\right|^{2}}{2 \log b},
$$

and if $\left|a_{2}\right| \leq-2 b \log b$, then $a_{3}$ can lie on the boundary of this disc. By elementary calculations one can show that the points of this circle for which the distance to the origin is extremal are

$$
A=\left\{1-b^{2}+\left(1+\frac{1}{\log b}\right)\left|a_{2}\right|^{2}\right\} \frac{a_{2}^{2}}{\left|a_{2}^{2}\right|^{2}},
$$

and

$$
B=\left\{-\left(1-b^{2}\right)+\left|a_{2}\right|^{2}\right\} \frac{a_{2}^{2}}{\left|a_{2}\right|^{2}} .
$$

These points are connected with the axial-symmetric extremum cases. The estimate due to this is

$$
\left|a_{3}\right| \leq \max (|A|,|B|),
$$

under the condition $\left|a_{2}\right| \leq-2 b \log b$. We rewrite this to obtain: If $a_{2}$ has a preassigned value for which $\left|a_{2}\right| \leq-2 b \log b$ then

$$
\left|a_{3}\right| \leq\left\{\begin{array}{l}
1-b^{2}+\left(1+\frac{1}{\log b}\right)\left|a_{2}\right|^{2}, \quad 0<b \leq e^{-1 / 2} \\
1-b^{2}-\left|a_{2}\right|^{2}, \quad e^{-1 / 2}<b<1
\end{array}\right.
$$

This inequality is sharp. The corresponding extremal domains are two-slit domains of the type indicate din Figure 10 (cases $\delta_{1}$ and $\delta_{3}$ ).

The second estimate is for $\operatorname{Re} a_{3}$. From (8.1) we obtain

$$
\operatorname{Re}\left\{\left(1+\frac{1}{2 \log b}\right) a_{2}^{2}\right\}-R \leqq \operatorname{Re} a_{3} \leq \operatorname{Re}\left\{\left(1+\frac{1}{2 \log b}\right) a_{2}^{2}\right\}+R
$$

If we write $a_{2}=u+i v$, the inequality reads

$$
u^{2}-\left(1+\frac{1}{\log b}\right) v^{2}-\left(1-b^{2}\right) \leq \operatorname{Re} a_{3} \leq\left(1+\frac{1}{\log b}\right) u^{2}-v^{2}+\left(1-b^{2}\right) .
$$

This inequality is sharp if $u^{2}+v^{2} \leq 4 b^{2} \log ^{2} b$.

In order to get an idea of the shape of the coefficient region $V_{3}$ we 


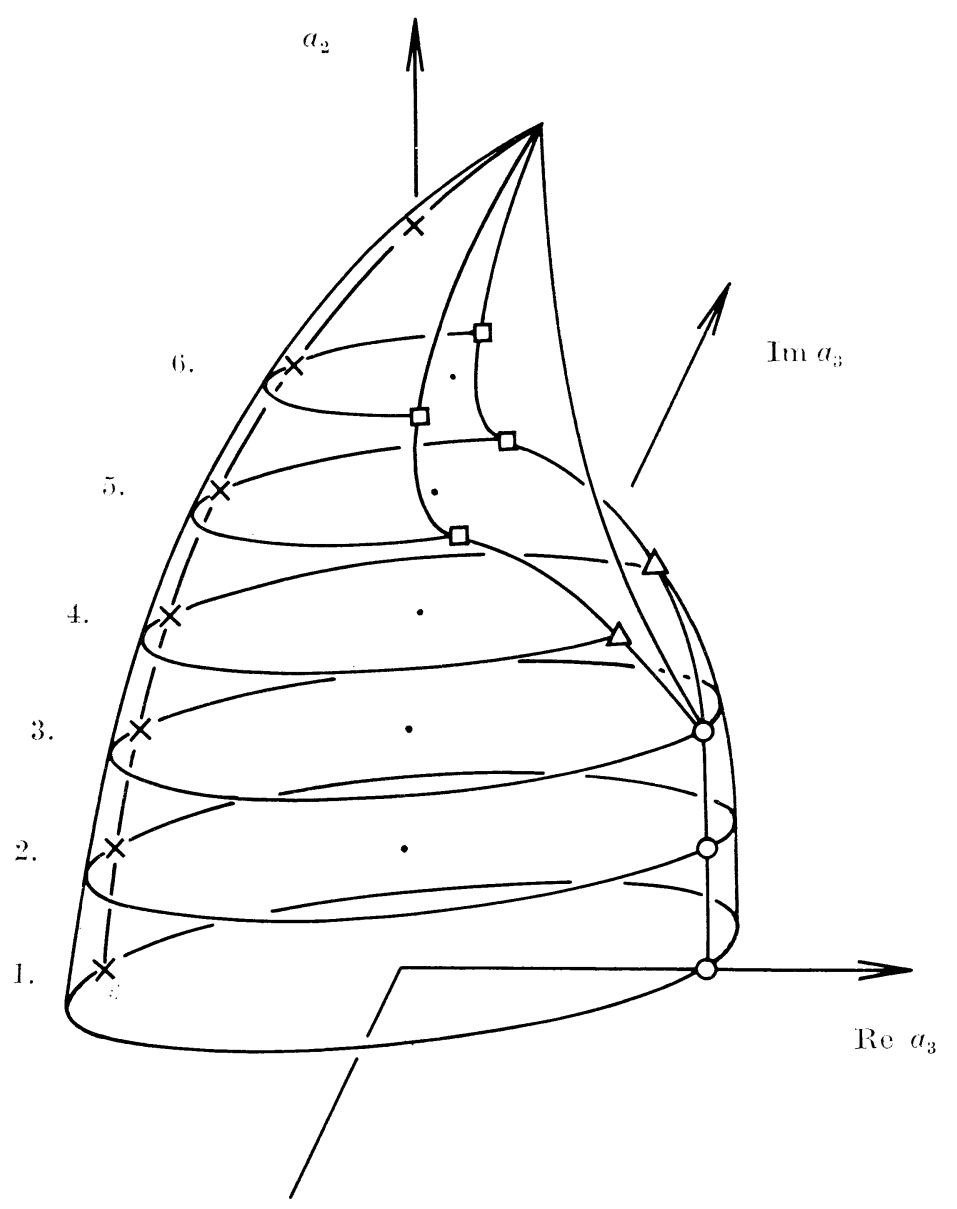

Figure 11.

determine two cross-sections. Take first $a_{2} \in R$. We investigate the range of $\left(a_{2}, \operatorname{Re} a_{3}, \operatorname{Im} a_{3}\right)$. For all values of $a_{2}$ with $\left|a_{2}\right| \leq-2 b \log b$, the disc (8.1) gives part of this range. If $\left|a_{2}\right|$ becomes greater, the function (7.9) is not always positive. However, $F$ is non-negative in a neighbourhood of the origin, so we obtain at least a part from the coefficient region. (In Section 3, using Löwner methods, we obtained the complete intersection with the plane $\left.\left(a_{2}, \operatorname{Re} a_{3}\right)\right)$. In those parts where $F<0$, the employed method fails to give sharp information about $V_{3}$. In Figures 11 and 12 we have indicated the different kind of extremal domains corresponding with different parts of the boundary of $V_{3}$. Some parts of $V_{3}$ are unknown.

It is also possible to make the restriction $a_{3} \in R$, and to investigate 

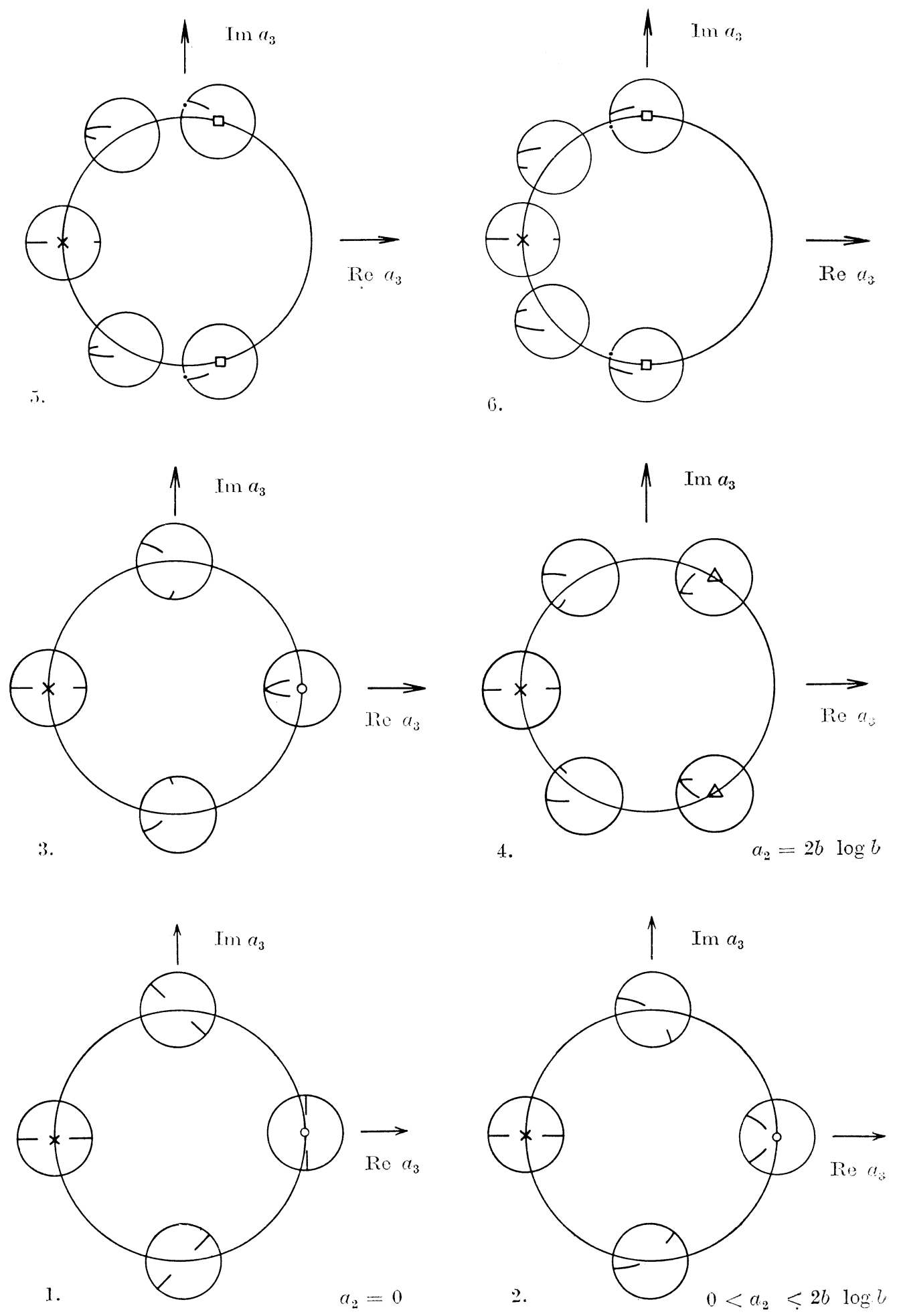

Figure 12. 
A. I. 592

the range of $\left(a_{3}, \operatorname{Re} a_{2}, \operatorname{Im} a_{2}\right)$. Substitution of $a_{2}=u+i v$ in (8.1) gives for the bounding surface the equation

$$
\begin{gathered}
a_{3}^{2}-2\left(1+\frac{1}{2 \log b}\right)\left(u^{2}-v^{2}\right) a_{3}+\left(1+\frac{1}{2 \log b}\right)^{2}\left(u^{2}+v^{2}\right) \\
-\left(1-b^{2}+\frac{u^{2}+v^{2}}{2 \log b}\right)^{2} \leq 0
\end{gathered}
$$

and this is sharp if $u^{2}+v^{2} \leq 4 b^{2} \log ^{2} b$.

University of Nijmegen

Nijmegen, the Netherlands

University of Helsinki

Helsinki, Finland 


\section{References}

[1] Charzyński, Z. - Janowski, W.: Domaine de variation des coefficients $A_{2}$ et $A_{3}$ des fonctions univalentes et bornées. - Bull. Soc. Sci. Lettres Loódź, 10 no 4 (1959) M.R. 23 no 3841.

[2] Peschl, E.: Zur theorie der schlichten Funktionen. - J. Reine Angew. Math. 176 (1936) pp. 61-96.

[3] Schaeffer, A. C. - Spencer, D. C.: Coefficient regions of schlicht functions. Amer. Math. Soc. Colloq. Publ. vol 35.

[4] Schiffer, M. - TaMmi, O.: A Green's inequality for the power matrix. - Ann. Acad. Sci. Fenn. Ser. A I 501.

[5] Tammi, O.: On the conformal mapping of symmetric schlicht domains. - Ibid. 173.

[6] - - - On optimizing parameters of the Power inequality for $a_{4}$ in the class of bounded univalent functions. - Ibid. 560. 